\title{
Consumo de macro e micronutrientes de idosos com diabetes mellitus tipo 2 atendidos no núcleo de apoio ao idoso
}

\author{
Consumption of macro and micronutrients of elderly people with type 2 \\ diabetes mellitus assisted at the nucleus to support for the elderly
}

Maria Goretti Pessoa de Araújo Burgos ${ }^{1}$, Eryka Maria dos Santos ${ }^{2}$, Andréia Albuquerque Cunha Lopes Morais $^{3}$, Priscilla Alves Santos ${ }^{4}$, Nathalia Caroline de Oliveira Melo ${ }^{5}$, Marcele Batista Madeiro Costa ${ }^{6}$

\begin{abstract}
RESUMO
Objetivo: Avaliar o consumo de macro e micronutrientes em idosos diabéticos atendidos no Núcleo de Atenção ao Idoso da Universidade Federal de Pernambuco (NAI/UFPE). Métodos: Estudo transversal com idosos saudáveis $(n=66)$ e com diabetes $(n=55)$ do NAI/UFPE. Obteve-se dados de prontuários e/ou questionários de frequência alimentar. Análise dietética realizada através do DietWin Professional 2008 e para estatística utilizou-se teste $t$-Student, admitindo-se nível de significância para $p<0,05$. Resultados: Os resultados mostraram a prevalência do sexo feminino em ambos os grupos (73,8\% no grupo saudável e $86,6 \%$ no grupo de idosos com diabetes), sendo idosos saudáveis com média de idade de $72,5 \pm 6,2$ anos, e grupo com diabetes com média de idade de $61,8 \pm 5,0$ anos $(p<0,05)$. 0 excesso de peso prevaleceu no grupo com diabetes (67\%) quando comparado ao grupo saudável $(49,2 \% ; p<0,027)$. A ingestão calórica dos pacientes com diabetes foi maior $(1257,94 \mathrm{kcal})$ em relação aos indivíduos saudáveis $(970,81 \mathrm{kcal} ; \mathrm{p}=0,01)$. O mesmo ocorreu para consumo de sódio $(1810,12 \mathrm{mg} \times 592,92 \mathrm{mg})$, cálcio $(721,44 \mathrm{mg} \times 286,23 \mathrm{mg})$, zinco $(8,73 \mathrm{mg} \times 6,26 \mathrm{mg})$, magnésio $(266,22 \mathrm{mg} \times 128,60 \mathrm{mg})$, vitamina A $(442,35 \mu \mathrm{g} \times 131,83 \mu \mathrm{g}) \mathrm{e}$ $\mathrm{E}(4,59 \mathrm{mg} \times 1,45 \mathrm{mg})(\mathrm{p}<0,01$ para todas as variáveis). Por outro lado, observou-se consumo inferior de potássio (1580,83 mg x 2349,23 mg; p<0,01) e proteína ( $9 \%$ x 17\%; p<0,01). Conclusão: Foi identificado um padrão alimentar hipercalórico nos idosos com diabetes, acompanhado de excesso de sódio, cálcio, magnésio e zinco, com reduzido consumo proteico e de potássio.
\end{abstract}

Palavras-chave: Idoso. Diabetes Mellitus. Consumo de Alimentos. Dieta.

\begin{abstract}
Objective: Evaluating the macro and micronutrient intake in elderly diabetic patients treated at the Elderly Care Center of the Federal University of Pernambuco, Brazil. Methods: A cross-sectional study was conducted with diabetic $(n=55)$ and non-diabetic $(n=66)$ older adults. Data were collected from patient charts and food frequency questionnaires. The analysis of diet was performed using the DietWin
\end{abstract}

1. Professora Postdoc do Departamento de Nutrição da Universidade Federal de Pernambuco (UFPE), Recife-PE.

2. Mestranda em Cirurgia pela UFPE, Recife-PE.

3. Mestre em Bioquímica e Fisiologia pela UFPE, Recife-PE.

4. Mestre em Nutrição pela UFPE, Recife-PE.

5. Mestranda em Nutrição pela UFPE, Recife-PE.

6. Especialista em Nutrição Clinica pelo Hospital das Clínicas da Universidade Federal de Pernambuco (HC-UFPE), Recife-PE.
CoRRESPONDÊnCIA:

Eryka Maria dos Santos Hospital das Clínicas da UFPE, Nutrição Av. Prof. Moraes Rego S/N. Cidade Universitária 50670-901 - Recife/PE - Brasil erykasantos.nutri@gmail.com

Recebido em: 08/08/2018 Aprovado em: 24/04/2019 
Professional 2008. The Student's t-test was used for the statistical analysis, with a $5 \%$ significance level. Results: The female sex accounted for $86.6 \%$ of the group with diabetes and $73.8 \%$ of the group without diabetes. Mean age was 61.8 years and 72.5 years, respectively. The excess weight was more frequent in the group with diabetes (67\%) when compared to the group without diabetes $(49.2 \%, \mathrm{p}<0.027)$. The caloric intake of patients with diabetes was higher $(1257.94 \mathrm{kcal})$ than in those without diabetes $(970.81 \mathrm{kcal}$, $\mathrm{p}=0.01)$. With the same statistical significance, we found the same for sodium (1810.12 $\mathrm{mg} \times 592.92 \mathrm{mg})$, calcium (721.44 mg x $286.23 \mathrm{mg})$, zinc ( $8.73 \mathrm{mg} \times 6.26 \mathrm{mg})$, magnesium (266.22 $\mathrm{mg}) \mathrm{mg} \times 128.60 \mathrm{mg})$, vitamin A (442.35 $\mathrm{g} \times 131.83 \mu \mathrm{g})$ and $\mathrm{E}(4.59 \mathrm{mg} \times 1.45 \mathrm{mg})$. On the other hand, lower potassium intake (1580.83 mg $\times 2349.23 \mathrm{mg}, \mathrm{p}<0.01)$ and protein $(9 \% \times 17 \%, \mathrm{p}<0.01)$ were observed. Conclusion: A hypercaloric eating pattern was identified in the elderly with diabetes, followed by an excess of sodium, calcium, magnesium, and zinc, with reduced protein and potassium consumption.

Keywords: Aged. Diabetes Mellitus. Food Consumption. Diet.

\section{INTRODUÇÃO}

O bem-estar físico e psicológico do idoso está diretamente relacionado à sua habilidade funcional. Dentro desse contexto, a nutrição desempenha importante papel na saúde desses indivíduos. Ao comparar idosos com adultos jovens, observa-se déficit de nutrientes, como ferro, cálcio, magnésio, potássio e zinco entre os indivíduos de maior faixa etária, com maior risco de desenvolver doenças carenciais. ${ }^{1,2,3}$ O desequilíbrio no consumo de nutrientes neste público é consequência de alterações psíquicas, somáticas e sociais. ${ }^{2}$ Estudos mostram que $76 \%$ da população brasileira acima dos 40 anos apresenta inadequação do consumo de micronutrientes essenciais, como cálcio, fósforo e magnésio. ${ }^{4}$

Um padrão dietético ocidentalizado, caracterizado por alto consumo de energia e de combustíveis energéticos, como carboidratos e gorduras saturadas, coincidindo com baixa ingestão de gorduras saudáveis e antioxidantes, contribui para a inflamação e desenvolvimento das doenças crônicas não transmissíveis, dentre elas o diabetes mellitus (DM) tipo $2 .{ }^{5}$

Dados de $2016^{6}$ confirmam o potencial protetor de um padrão alimentar baseado em vegetais na prevenção primária do diabetes, em particular entre indivíduos de meia-idade, provavelmente pelo teor reduzido de carboidratos e elevada presença de micronutrientes. No entanto, o impacto que o estilo de vida tem no curso da resistência à insulina depende da adesão individual.

A prescrição da dieta balanceada e individualizada é imprescindível e deve ser realizada considerando-se aspectos nutricionais, socioeconômicos, culturais e psicológicos do indivíduo. Além dos determinantes sociais, o envelhecimento e a presença de diabetes também podem influenciar direta ou indiretamente na escolha da qualidade do alimento. As alterações corporais e o estado nutricional próprios do envelhecimento, além da presença de Doenças Crônicas não transmissíveis (DCNT), comprometem o estado de saúde e a necessidade nutricional do indivíduo idoso.7 Quando se trata de idosos portadores de DM, a literatura é escassa, tanto a nível nacional, quanto internacional. Porém, estudo realizado no Japão que avaliou 207 idosos diabéticos, mostrou que o consumo adequado de vitamina B2, pantotenato, cálcio e vegetais podem ajudar a prevenir o declínio cognitivo em homens idosos com diabetes mellitus, reforçando a importância de uma nutrição adequada tanto em macro como em micronutrientes para essa faixa etária. ${ }^{8}$

O objetivo desse trabalho foi identificar aspectos alimentares de macro e micronutrientes em idosos diabéticos.

\section{MÉTODOS}

Estudo transversal do tipo série de casos, realizado no período de janeiro a dezembro de 2015, com idosos saudáveis (IS) e idosos portadores de diabetes mellitus tipo 2 (DM), selecionados por conveniência no Núcleo de Apoio ao Idoso da Universidade Federal de Pernambuco (NAI/UFPE), que atende em média 354 idosos, em todas as especialidades de saúde (2018). Participaram da pesquisa aqueles que concordaram e, posteriormente assinaram um termo de consentimento. 
Como critério de inclusão o idoso portador de DM deveria ter um tempo > cinco anos de doença, com diagnóstico realizado a partir de Hemoglobina A1c $\leq 8 \%$, duas ou mais glicemias de jejum $\geq 126 \mathrm{mg} / \mathrm{dL}$ ou glicemia ao acaso $\geq 200 \mathrm{mg} / \mathrm{dL}^{9}$. Foram considerados aqueles com idade $\geq 60$ anos, de ambos os sexos, orientados, sem amputação ou patologias que provocassem alterações na composição corporal, que deambulassem normalmente sem ajuda de terceiros, e que estivessem em uso de hipoglicemiante $1 / 2 x$ dia. Não foram considerados idosos com diagnóstico recente, uma vez que poderíamos ter inclusão de falsos positivos, além de indivíduos sem uso diário de hipoglicemiante oral. Foram coletados dados demográficos diretamente com o paciente e, no mesmo momento, realizado as demais avaliações.

A avaliação nutricional foi realizada através de métodos diretos (Índice de Massa Corporal-IMC) e indiretos (questionário de frequência alimentar QFA - dos últimos 6 meses). O peso corporal foi aferido em balança de marca WELMY $®$, com capacidade de $150 \mathrm{~kg}$, e a altura mensurada por meio do estadiômetro acoplado a balança. Calculou-se o IMC dividindo-se o peso corporal $(\mathrm{kg})$ pelo quadrado da altura $(\mathrm{m})$. Os pontos de corte adotados para classificação nutricional atenderam as recomendações propostas por Lipschitz ${ }^{10}$ para indivíduos $\geq 60$ anos. Para avaliação do consumo foi utilizado um questionário de frequência alimentar qualitativo. ${ }^{11}$

A análise da composição da dieta foi realizada pelo cálculo do consumo de calorias totais, carboidratos, proteínas, lipídeos, vitaminas A, C e E, ferro (Fe), cálcio $(\mathrm{Ca})$, sódio $(\mathrm{Na})$, zinco $(\mathrm{Zn})$ e magnésio $(\mathrm{Mg})$, utilizando-se o programa DietWin Professional 2008. Foi aplicado o teste de Kolmogorov-Smirnov para avaliar a normalidade da distribuição das variáveis e, todas apresentaram distribuição Gaussiana. A análise estatística foi realizada entre os grupos, com os dados apresentados em média e desvio padrão através do teste t-Student na comparação entre os 2 grupos de idosos, considerando estatisticamente significante quando $p<0,05$.

A pesquisa foi iniciada após aprovação do Comitê de Ética em Pesquisa envolvendo seres humanos do Centro de Ciências da Saúde da Universidade Federal de Pernambuco sob o n. 0 38255214.5.0000.5208.

\section{RESULTADOS}

Este estudo envolveu 121 idosos não institucionalizados, os quais foram distribuídos em dois grupos, sendo 66 saudáveis (IS) e 55 portadores de DM tipo 2 (DM). Todos tiveram a frequência alimentar avaliada nos últimos seis meses. No grupo dos IS, $73,8 \%$ eram mulheres, com média de idade de $72,5 \pm 6,2$ anos. A maioria domiciliada na área urbana $(96,9 \%)$, sendo todos aposentados com renda familiar entre 1-3 salários mínimos. Os DM apresentavam diagnóstico da doença por tempo $\geq 5$ anos (5-28 anos), com maioria mulheres $(86,6 \%)$, na faixa etária de 61,8 anos (60-85 anos), residentes no Recife/região metropolitana. Quanto aos dados antropométricos, entre os idosos saudáveis $49,2 \%$ apresentavam excesso de peso, enquanto no grupo DM $67 \%$ estavam nesse IMC (Tabela 1 ).

\section{Tabela 1}

Características sociodemográficas em idosos com diabetes mellitus tipo 2 e idosos sadios. Recife-PE, Brasil, 2015

\begin{tabular}{lcc}
\hline Variáveis & $\begin{array}{c}\text { Diabéticos } \\
(n=55)\end{array}$ & $\begin{array}{c}\text { Sadios } \\
(n=66)\end{array}$ \\
\hline Sexo & $F 86,6 \%$ & $F 73,8 \%$ \\
Média de idade & 61,8 anos & 72,5 anos \\
Procedência & Urbana: $96,9 \%$ & Urbana: $95,5 \%$ \\
$\begin{array}{l}\text { R Excesso de } \\
\text { peso }\end{array}$ & $67,0 \%$ & Rural: $4,5 \%$ \\
\hline
\end{tabular}

Fonte: Produção dos autores.

O consumo calórico dos DM foi significativamente maior do que o dos IS $(1257,94 \mathrm{kcal} \pm$ $363,12$ versus $970,8 \mathrm{kcal} \pm 196,40 ; p<0,01)$. Também houve diferença na distribuição de proteína, que no grupo IS foi $17 \%$ e no grupo DM, apenas $9 \%$ $(p<0,01)$ (Tabela 2).

Quanto à ingestão de minerais, detectou-se semelhança entre o Fe (DM: 4,60 mg $\pm 2,61$; IS: $4,94 \mathrm{mg} \pm 1,45 ; \mathrm{p}<0,39)$ nos dois grupos. Por outro lado, observou-se diferenças estatisticamente significantes para $\mathrm{Na}$ (DM: $1810,12 \mathrm{mg} \pm$ 1430,29 versus IS: $592,92 \mathrm{mg} \pm 150,58$; $\mathrm{p}<$ 0,01), $\mathrm{Ca}$ (DM: 721,44 mg \pm 407,03 versus IS: $286,23 \mathrm{mg} \pm 75,75 ; \mathrm{p}<0,01)$, Zn (DM: $8,73 \mathrm{mg} \pm 4,14$ versus IS: $6,26 \mathrm{mg} \pm$ 3,37; $\mathrm{p}<0,01)$ e $\mathrm{Mg}$ (DM: $266,22 \mathrm{mg} \pm 84,44$ versus IS: $128,60 \mathrm{mg} \pm 46,89 ; \mathrm{p}<0,01)$, 
com os DM apresentando maior consumo, enquanto 0 K (DM: $2349,23 \mathrm{mg} \pm 690,17$ versus IS: $1580,83 \mathrm{mg} \pm$ $364,24 ; p<0,01)$ foi mais consumido pelos saudáveis (Tabela 2).

Com relação às vitaminas antioxidantes, apresentadas na Tabela 2, houve um maior con- sumo de vitamina A (DM: $442,35 \mu \mathrm{g} \pm 317,60$ versus IS: $131,83 \mu \mathrm{gg} \pm 65,34 ; \mathrm{p}<0,01)$ e E (DM: $4,59 \mathrm{mg} \pm 3,73$ versus IS: $1,45 \mathrm{mg} \pm 0,96$; $\mathrm{p}<0,01)$ nos idosos com diabetes, o que não ocorreu em relação a vitamina C (DM: $112,14 \mathrm{mg} \pm$ $106,53$ versus IS: $128,26 \mathrm{mg} \pm 67,22 ; \mathrm{p}<0,34)$.

Tabela 2

Consumo de macro e micronutrientes de idosos com diabetes mellitus tipo 2 e idosos sadios. Recife-PE, Brasil, 2015

\begin{tabular}{lcccc}
\hline & $\begin{array}{c}\text { Valor de } \\
\text { referência/diária }\end{array}$ & $\begin{array}{c}\text { Consumo Diabéticos } \\
(\mathrm{n}=55)\end{array}$ & $\begin{array}{c}\text { Consumo Saudáveis } \\
(\mathrm{n}=66)\end{array}$ & Valor de $p$ \\
\hline Calorias totais (Kcal) & - & $1257,94 \pm 363,12$ & $970,80 \pm 196,40$ & $<0,01^{*}$ \\
Carboidratos (\%) & $45-60^{* *}$ & 62,00 & 62,00 & $0,90 \bullet$ \\
Proteínas (\%) & $15-20 * *$ & 9,00 & 17,00 & $<0,01^{*}$ \\
Lipídios (\%) & $20-35^{* *}$ & 29,00 & 21,00 & $0,26 \bullet$ \\
Vitamina A ( $\mu \mathrm{g})$ & $800^{* * *}$ & $442,35 \pm 317,60$ & $131,83 \pm 65,34$ & $<0,01^{*}$ \\
Vitamina C (mg) & $62,5^{* * *}$ & $1121,14 \pm 106,53$ & $128,26 \pm 67,22$ & $<0,34$ \\
Vitamina E (mg) & $12^{* * *}$ & $4,59 \pm 3,73$ & $1,45 \pm 0,96$ & $<0,01^{*}$ \\
Ferro (mg) & $5,5 * * *$ & $4,60 \pm 2,61$ & $4,94 \pm 1,45$ & $<0,39$ \\
Sódio (mg) & $1.200^{* * *}$ & $1810,12 \pm 1430,29$ & $592,92 \pm 150,58$ & $<0,01^{*}$ \\
Cálcio (mg) & $1.000^{* * *}$ & $721,44 \pm 407,03$ & $286,23 \pm 75,75$ & $<0,01^{*}$ \\
Zinco (mg) & $8,1 * * *$ & $8,73 \pm 4,14$ & $6,26 \pm 3,37$ & $<0,01^{*}$ \\
Magnésio (mg) & 307,5 & $266,22 \pm 84,44$ & $128,60 \pm 46,89$ & $<0,01^{*}$ \\
Potássio (mg) & 4700 & $2349,23 \pm 690,17$ & $1580,83 \pm 364,24$ & $<0,01^{*}$ \\
\hline
\end{tabular}

Fonte: Produção dos autores.

Teste t-Student* e Teste qui-quadrado de Pearson•-Valores de $p$ considerados estatisticamente significantes $(p<0,05)$.

**Sociedade Brasileira de Diabetes (2017).

***Dietary reference intakes 1997 Magnésio; 2000 para vitaminas E, C; 2001 para vitamina A, zinco e ferro; 2005 Sódio e Potássio; 2011 Cálcio - Valores para ambos os sexos calculados pela média da recomendação homem/mulher.

\section{DISCUSSÃO}

No presente estudo, a casuística foi composta principalmente por mulheres $(73,8 \%)$ refletindo o interesse desse grupo na atenção à saúde, o que também é observado na atenção primária à saúde. Tais evidências corroboram com os achados da literatura que identificam uma maior demanda feminina por serviços de saúde. ${ }^{12}$

Quanto aos dados antropométricos, O IMC evidenciou prevalência de excesso de peso nos DM, quando comparados ao grupo IS. Estudo de Lira et al. ${ }^{13}$, ao avaliar adultos e idosos atendidos em hospital universitário desta mesma cidade, também encontraram percentual elevado de excesso de peso pelo IMC. Esta medida apesar de apresentar desvantagens quando comparada as demais que avaliam composição corporal, é ainda a mais aceita universalmente, para categorizar o sobrepeso e a obesidade. ${ }^{14}$
Seu princípio básico é expressar a gordura corporal em excesso, quantificando a obesidade global. ${ }^{15}$

Apesar da fácil aplicabilidade, a utilização do IMC em idosos gera controvérsias. Segundo Castro, o processo de envelhecimento é influência importante no aumento do tecido adiposo na região abdominal, uma vez que os idosos sofrem alterações na composição corporal como fator natural de senescência e/ou devido à ocorrência de distúrbios metabólicos. Essas alterações levam à redistribuição da gordura dos membros para o tronco, resultando em maior adiposidade visceral, sendo este processo mais significativo em mulheres na pós-menopausa do que em homens. ${ }^{16}$ Sabe-se que isso ocorre em todas as idades, principalmente devido à gordura essencial, que no sexo feminino é, aproximadamente, quatro vezes superior à dos indivíduos do sexo masculino. ${ }^{17}$

A condição de excesso de peso relacionada diretamente ao DM observada no presente estudo também foi verificada por Gomes et al. ${ }^{18}$, no qual 
verificaram que dos 2.519 pacientes adultos com diabetes avaliados, $76 \%$ deles se encontravam com sobrepeso ou obesidade. Outro estudo realizado em Curitiba-PR, com 1.069 mulheres idosas, identificou $43,5 \%$ delas com sobrepeso e $34 \%$ em condição de obesidade. ${ }^{19}$ Barceló et al. ${ }^{20}$ também verificaram forte associação da prevalência de diabetes com o IMC elevado e o excesso de peso foi mais comum nas mulheres, por possuírem maior quantidade de gordura corporal e valores de massa corporal e estatura inferiores aos homens (40,5\%).

A literatura reconhece a obesidade como um fator de risco à incidência de doenças, inclusive do DM e recomenda redução e/ou controle do peso, como uma das principais estratégias de tratamento não farmacológico do diabetes. ${ }^{21}$ Silveira et al. ${ }^{22}$ mostraram alta prevalência de obesidade abdominal em idosos, principalmente em mulheres, e sua associação com hipertensão, doenças respiratórias e DM. Atrelados a esse contexto, a Sociedade Brasileira de Diabetes (SBD) relata que a diminuição de 5 a $10 \%$ do peso corporal poderia reduzir os níveis de glicemia, adiar a progressão da doença, reduzir as necessidades insulínicas e, inclusive nos casos iniciais, permitiria retirar o tratamento farmacológico. ${ }^{9}$

Quanto ao consumo alimentar, ficou evidenciado no grupo de indivíduos com DM um consumo calórico significativamente maior quando comparado ao grupo dos IS. Resultados que poderiam ser explicados pela hiperfagia observada na maioria dos DM, além da preocupação de estarem bem alimentos a fim de evitar hipoglicemia. ${ }^{23}$ No presente estudo, $98 \%$ dos DM apresentavam hiperfagia, em algum momento do dia, mesmo com controle glicêmico adequado.

Ao mesmo tempo, observa-se no Brasil modificações ocorridas no consumo alimentar dos idosos nos últimos anos, com maior consumo alimentar de calorias, como gorduras, alimentos açucarados e refinados. ${ }^{12,} 24,25$ No entanto, a escassez de estudos que avaliem o consumo alimentar nessa população, impossibilita a comparação entre os resultados. ${ }^{25}$

Os achados evidenciam uma inadequação no consumo de macronutrientes, com menor consumo proteico e maior consumo de lipídio pelo grupo DM, fator preocupante uma vez que é recomendado consumo entre $15-20 \%$ de proteína. ${ }^{9}$ Pesquisas avaliando a proporção de macronutrientes da alimentação de idosos saudáveis têm encontrado variações seme-
Ihantes, de $12 \%$ a $18 \%$ para proteína, $44 \%$ a $60 \%$ para carboidratos e $25 \%$ a $42 \%$ para gordura. ${ }^{26,27}$

Estudo avaliando consumo de idosos saudáveis em todas as regiões do Brasil também mostrou inadequação no consumo calórico e de macronutrientes, sendo a proteína o macronutriente que apresentou maior adequação, diferente do baixo consumo de carboidrato e alto consumo de lipídios ${ }^{28}$, resultados opostos ao detectado neste estudo.

Essa inadequação no consumo alimentar também foi evidenciada por estudo realizado por Zanchim et al. ${ }^{29}$, que avaliaram 100 indivíduos adultos ou idosos com DM e mostraram que apesar do consumo frequente de leite, frutas e salada crua, os legumes foram os marcadores saudáveis menos consumidos e foi alto o consumo de alimentos ultraprocessados, com percentual de $67 \%$ para biscoito salgado, por exemplo.

A Organização Mundial de Saúde $(\mathrm{OMS})^{30}$ recomenda para adultos saudáveis intervalos de distribuição aceitáveis dos macronutrientes, sendo de $10 \%$ a $15 \%$ para proteína, de $55 \%$ a $75 \%$ de carboidratos e $15 \%$ a $30 \%$ de lipídios. Avaliando os valores médios da proporção de macronutrientes da alimentação dos idosos saudáveis deste estudo, é possível observar que os mesmos foram semelhantes ao preconizado pelo OMS. Entretanto, no grupo DM, o consumo de carboidratos e proteínas corrobora com a recomendação proposta pela SBD (2017) ${ }^{9}$, com indicativos de 45 a $60 \%$ e 15 a $20 \%$, respectivamente. O consumo de lipídios nesse grupo não excedeu a recomendação, entretanto, tanto nos idosos saudáveis quanto naqueles com diabetes, os resultados mostraram o maior consumo de gordura saturada, ocasionado principalmente pelo consumo de margarina, justificado pelo baixo custo.

Quanto à ingestão de minerais, foi observado semelhança entre o consumo de $\mathrm{Fe}$ nos dois grupos. Por outro lado, o grupo DM mostrou maior consumo de $\mathrm{Na}, \mathrm{Ca}, \mathrm{Zn}$ e $\mathrm{Mg}$, enquanto o $\mathrm{K}$ foi mais consumido pelos idosos saudáveis. Na avaliação de recomendações atuais para diabéticos, observa-se necessidade de menor ingestão de $\mathrm{Na}$ e maior de $\mathrm{K}$, exatamente o oposto do encontrado no grupo DM, apesar do consumo estar na faixa dos valores recomendados. ${ }^{9}$ Quando comparados aos valores de referência estabelecidos pela Dietary Reference Intakes (DRIs) ${ }^{31-35}$, ambos os grupos apresentaram baixo consumo de minerais, apenas o $\mathrm{Na}$ no grupo 
DM apresentou-se elevado em relação à recomendação diária nessa fase da vida.

O INTERSALT é considerado o mais importante estudo epidemiológico multicêntrico, que avaliou 52 populações em 32 diferentes países e, mostrou que as comunidades que consumiam mais sal tinham maior incremento da pressão com o envelhecimento. ${ }^{36} \mathrm{Em}$ relação ao K, o National High Blood Education Program, afirmou que o $\mathrm{K}$ auxilia na redução do peso e controle da pressão arterial. ${ }^{37}$

Com relação às vitaminas antioxidantes, houve um maior consumo de vitamina $A$ e $E$ nos idosos com DM, dado altamente benéfico, o que não ocorreu em relação à vitamina $C$. Os dados na literatura relacionados ao consumo vitamínico por este grupo ainda são muito escassos, impossibilitando a comparação deste consumo. Vitaminas antioxidantes reduzem a peroxidação lipídica, a oxidação das partículas de LDL-colesterol e melhoram a função endotelial e a vasodilatação dependente do endotélio. ${ }^{38} \mathrm{~A}$ suplementação com antioxidantes, como vitaminas $A, E, C$ quando não ocorre deficiência no consumo, não é recomendada por falta de evidências científicas que mostrem benefícios. ${ }^{7}$

Indivíduos maiores que 60 anos apresentam multiplicidade de patologias e, portanto, consomem maior número de medicamentos de uso crônico. $\mathrm{O}$ uso de diferentes fármacos, nesta fase da vida, tem deixado de ser esporádico tornando-se habitual. A polifarmácia em idosos aumenta a incidência de efeitos colaterais e interações droga-nutrientes, prejudicando significativamente a absorção de nutrientes antioxidantes. Nesse sentido, esta utilização em longo prazo interfere na digestão, na absorção e no metabolismo de nutrientes. ${ }^{7}$

Entretanto, nosso estudo possui limitações. A amostra não foi representativa da população geral, visto que a seleção foi restrita a idosos hígidos, não acamados que praticam atividade física regularmente e residem na área metropolitana do Recife/PE.

\section{CONCLUSÃO}

O padrão alimentar dos DM idosos é caracterizado por maior consumo calórico, com inadequação de macro e micronutrientes. A literatura avaliando o consumo alimentar em idosos com dia- betes mellitus tipo 2 é escassa e, portanto, destaca-se a importância do profissional nutricionista em realizar atendimento individualizado e periódico nessa faixa etária com DCNT.

\section{REFERÊNCIAS}

1. Valls $\mathrm{T}$, Mach N. Risk of malnutrition in people older than 75 years. Med Clin (Barc). 2012;139(4):157-160. doi: 10.1016/j.medcli.2012.01.029

2. Brownie S. Why are elderly individuals at risk of nutritional deficiency? Int J Nurs Pract. 2006;12(2):110-118. doi: $10.1111 /$ j.1440-172X.2006.00557.x

3. Venturini CD, Engroff P, Sgnaolin V, Carli GA, Morrone FB, Silva Filho IG, et al. Consumo de nutrientes em idosos residentes em Porto Alegre (RS), Brasil: um estudo de base populacional. Cienc Saude Colet. 2015;20(12):3701-11. Disponível em: http://www.scielo.br/pdf/csc/v20n12/ 1413-8123-csc-20-12-3701.pdf

4. Pinheiro MM, Schuch NJ, Genaro PS, Ciconelli RM, Ferraz MB, Martini LA. Nutrient intakes related to osteoporotic fractures in men and women: The Brazilian Osteoporosis Study (BRAZOS). Nutr J. 2009;8:6. doi: 10.1186/1475-2891-8-6

5. Calton EK, James AP, Pannu PK, Soares MJ. Certain dietary patterns are beneficial for the metabolic syndrome: reviewing the evidence. Nutr Res. 2014;34(7):559-68. doi: $10.1016 /$ j.nutres.2014.06.012

6. Koloverou E, Panagiotakos DB, Georgousopoulou EN, Grekas A, Christou A, Chatzigeorgiou M, et al. Dietary Patterns and 10-year (2002-2012) Incidence of Type 2 Diabetes: Results from the ATTICA Cohort Study. Rev Diabet Stud. 2016;13(4):246-56. doi: 10.1900/ RDS.2016.13.246

7. Campos MTFS, Monteiro JBR, Ornelas APRC. Fatores que afetam o consumo alimentar e a nutrição no idoso. Rev Nutr. 2000;13(3):157-65. Disponível em: http://www.scielo.br/pdf/rn/v13n3/7902.pdf

8. Araki A, Yoshimura $Y$, Sakurai T, Umegaki H, Kamada C, Iimuro $S$, et al. Low intakes of carotene, vitamin B2, pantothenate and calcium predict cognitive decline among elderly patients with diabetes mellitus: the Japanese elderly diabetes intervention trial. Geriatr Gerontol Int. $2017 ; 17(8)$ :1168-75. doi: 10.1111/ggi.12843

9. Oliveira JEP, Montenegro Junior RM, Vencio S, Foss-Freitas MC, organização. Diretrizes da Sociedade Brasileira de Diabetes 2017-2018. São Paulo: Editora Clannad; 2017.

10. Lipschitz, DA. Screening for nutritional status in the elderly. Prim Care. 1994;21(1):55-67.

11. Salvo VLMA, Gimeno SGA. Reprodutividade e validade do questionário de frequência e consumo de alimentos. Rev Saúde Pública. 2002;36(4):505-12. Disponível em: http://www.scielo.br/pdf/rsp/v36n4/11771.pdf 
12. Pimentel IRS, Coelho BC, Lima JC, Ribeiro FG, Sampaio FPC, Pinheiro RP, et al. Caracterização da demanda em uma Unidade de Saúde da Família. Rev Bras Med Fam. 2011;6(20):175-81. dOI: 10.5712/rbmfc6(20)95

13. Lira MCA, Souza NMM, Burgos MGPA. Prevalência de fatores de risco para doenças cardiovasculares em diabéticas. Nutr Clin Diet Hosp. 2017;37(1):75-81. doi: 10.12873/371mayaramenezes

14. World Health Organization. Obesity: Preventing and Managing the Global Epidemic. Geneva: WHO; 2000.

15. Nevill AM, Stavropoulos-Kalinoglou A, Metsios GS, Koutedakis $Y$, Holder RL, Kitas GD, et al. Inverted BMI rather than BMI is a better proxy for percentage of body fat. Annals of Biology Human. 2011;38(6):681-4. doi: $10.3109 / 03014460.2011 .606832$

16. Wang Q, Hassager C, Ravn P, Wang S, Christiansen C. Total and regional body composition change in early postmenopausal women: age-related or menopauserelated? Am J Clin Nutr. 1994;60(6):843-8. doi: 10.1093/ajcn/60.6.843

17. Lebrão $M L$, Laurenti R. Saúde, bem-estar e envelhecimento: o estudo SABE no Município de São Paulo. Rev Bras Epidemiol. 2005;8(2):127-41. Disponível em: http://www.scielo.br/pdf/rbepid/v8n2/05.pdf

18. Gomes MB, Giannella Neto D, Mendonça E, Tambascia $M A$, Fonseca RM, Réa RR, et al. Prevalência de sobrepeso e obesidade em pacientes com diabetes mellitus do tipo 2 no Brasil: estudo multicêntrico nacional. Arq Bras Endocrinol Metab. 2006;50(1):136-144. Disponível em: http://www.scielo.br/pdf/abem/v50n1/28735.pdf

19. BuzzacheraCF,KrauseMP, ElsangedyHM, HallageT,GranatoP, Krinski K, et al. Prevalência de sobrepeso e obesidade geral e central em mulheres idosas da cidade de Curitiba, Paraná. Rev Nutr. 2008;21(5):525-533. Disponível em: http://www.scielo.br/pdf/rn/v21n5/a05v21n5.pdf

20. Barceló A, Pelaez M, Rodriguez-Wong L, Pastor-Valero M. The prevalence of diagnosed diabetes among the elderly of seven cities in Latin America and the Caribbean: The Health, Wellbeing, and Aging (SABE) Project. J Aging Health. 2006;18(2):224-239. doi: 10.1177/0898264305285728

21. Roediger MA, Marucci MFN, Gobbo LA, Dourado DAQS, Santos JLF, Duarte YAO, et al. Diabetes mellitus referida: incidência e determinantes, em coorte de idosos do município de São Paulo, Brasil, Estudo SABE-Saúde, Bem-Estar e Envelhecimento. Cien Saude Colet. 2018;23:3913-22. doi: $10.1590 / 1413-812320182311.13062016$

22. Silveira EA, Vieira LL, Souza JD. Elevada prevalência de obesidade abdominal em idosos e associação com diabetes, hipertensão e doenças respiratórias. Cien Saude Colet. 2018;23:903-12. doi: $10.1590 / 1413-81232018233.01612016$

23. Moura LP, Gomes RJ, Leme JA, Voltarelli FA, Ribeiro C, de Moura $R F$, et al. Insulina pancreática de ratos diabéticos tipo 1 submetidos a um protocolo de treinamento físico individualizado. Motricidade. 2012;8(1):23-32. doi: $10.6063 /$ motricidade.8(1).234

24. Popkin BM. The nutrition transition and obesity in the developing world. J Nutr. 2001;131(3):871-873. doi: 10.1093/jn/131.3.871S
25. Amado TCF, Arruda IKG, Ferreira RAR. Aspectos alimentares, nutricionais e de saúde de idosas atendidas no Núcleo de Atenção ao Idoso - NAI, Recife/ 2005. ALAN. 2007;57(4):366-372. Disponível em: https://www.alanrevista.org/ediciones/2007/4/art-9/

26. Fulgoni VL. Current protein intake in America: analysis of the National Health and Nutrition Examination Survey, 2003-2004. Am J Clin Nutr. 2008;87(5):1554-7. doi: 10.1093/ajcn/87.5.1554S

27. García-Arias MT, Villarino Rodrígues A, García-Linares MC, Rocandio AM, García-Fernández MC. Daily intake of macronutrients in a group of institutionalized elederly people in León. Spain. Nutr Hosp. 2003;18(2):87-90.

28. Previdelli AN, Goulart RMM, Aquino RC. Balanço de macronutrientes na dieta de idosos brasileiros: análises da Pesquisa Nacional de Alimentação 2008-2009. Rev Bras Epidemiol. 2017;20(1):70-80. doi: 10.1590/19805497201700010006

29. Zanchim MC, Kirsten VR, Marchi ACB. Marcadores do consumo alimentar de pacientes diabéticos avaliados por meio de um aplicativo móvel. Cien saude colet. 2018;23(12):4199-208. doi: 10.1590/1413812320182312.01412017

30. Brasil. Ministério da Saúde. Secretaria de Atenção à Saúde. Departamento de Atenção Básica. Guia alimentar para a população brasileira: promovendo a alimentação saudável. Brasília (DF): Ministério da Saúde; 2008.

31. Institute of Medicine. Dietary reference intakes for calcium, phosphorus, magnesium, vitamin $\mathrm{D}$, and fluoride. Washington (DC): National Academy Press (US); 1997.

32. Monsen ER. Dietary reference intakes for the antioxidant nutrients: Vitamin C, vitamin E, selenium, and carotenoids. American Dietetic Association. Journal of the American Dietetic Association. 2000;100(6):637-40.

33. Trumbo $P$, Yates $A A$, Schlicker $S$, Poos $M$. Dietary reference intakes for vitamin $\mathrm{A}$, vitamin $\mathrm{K}$, arsenic, boron, chromium, copper, iodine, iron, manganese, molybdenum, nickel, silicon, vanadium, and zinc. Washington (DC): National Academies Press (US); 2001.

34. Institute of Medicine. Dietary reference intakes for energy, carbohydrate, fiber, fat, fatty acids, cholesterol, protein, and amino acids. Washington (DC): National Academies Press (US); 2005.

35. Del Valle HB, Yaktine AL, Taylor CL, Ross AC, editors. Dietary reference intakes for calcium and vitamin D. Washington (DC): National Academies Press (US); 2011.

36. Intersalt: an international study of electrolyte excretion and blood pressure. Results for 24 hour urinary sodium and potassium excretion. Intersalt Cooperative Research Group. Br Med J. 1998;297:319-28. doi: 10.1136/bmj.297.6644.319

37. National Hight Blood Pressure Education Program Working (NHBPEP) Groups Report on Hypertension in the Elderly. Hypertension. 1994;23(3):275-85.

38. Cuerda C, Luengo LM, Valero MA, Vidal A, Burgos R, Calvo $\mathrm{FL}$, et al. Antioxidantes y diabetes mellitus: revisión de la evidencia. Nutr Hosp. 2011;26(1):68-78. Disponível em: http://scielo.isciii.es/pdf/nh/v26n1/ revision_4.pdf 